\title{
ON CARLESON MEASURES INDUCED BY BELTRAMI COEFFICIENTS BEING COMPATIBLE WITH FUCHSIAN GROUPS
}

\author{
Shengjin Huo \\ Tiangong University, Department of Mathematics \\ Tianjin 300387, P. R. China; huoshengjin@tjpu.edu.cn
}

\begin{abstract}
Let $\mu$ be a Beltrami coefficient on the unit disk, which is compatible with a finitely generated Fuchsian group $G$ of the second kind. In this paper we show that if $\frac{|\mu|^{2}}{1-|z|^{2}} d x d y$ satisfies the Carleson condition on the infinite boundary of the Dirichlet fundamental domain of $G$, then $\frac{|\mu|^{2}}{1-|z|^{2}} d x d y$ is a Carleson measure on the unit disk.
\end{abstract}

\section{Introduction}

A Fuchsian group is a discrete Möbius group $G$ acting on the unit disk $\Delta$. A Fuchsian group is said to be of the first kind if its limit set is the entire circle and of the second kind otherwise. A Fuchsian group $G$ is called cocompact if $\Delta / G$ is compact and is called convex cocompact if $G$ is finitely generated without parabolic elements. All cocompact groups are first kind and convex cocompact groups minus cocompact groups are second kind. A Fuchsian group $G$ is of divergence type if

$$
\Sigma_{g \in G}(1-|g(0)|)=\infty \quad \text { or } \quad \sum_{g \in G} \exp (-\rho(0, g(0)))=\infty
$$

where $\rho(0, g(0))$ is the hyperbolic distance between 0 and $g(0)$. Otherwise, we say that it is of convergence type. All second kind groups are of convergence type. For more details about Fuchsian groups, see [9].

For $g$ in $G$, we denote by $\mathcal{D}_{z}(g)$ the closed hyperbolic half-plane containing $z$, bounded by the perpendicular bisector of the segment $[z, g(z)]_{h}$. The Dirichlet fundamental domain $\mathcal{F}_{z}(G)$ of $G$ centered at $z$ is the intersection of all the sets $\mathcal{D}_{z}(g)$ with $g$ in $G-\{i d\}$. For simplicity, in this paper we use the notation $\mathcal{F}$ for the Dirichlet fundamental domain $\mathcal{F}_{z}(G)$ of $G$ centered at $z=0$.

A positive measure $\lambda$ defined in a simply connected domain $\Omega$ is called a Carleson measure if there exists some constant $\mathrm{C}$ which is independent of $r$ such that, for all $0<r<\operatorname{diameter}(\partial \Omega)$ and $z \in \partial \Omega$,

$$
\lambda(\Omega \cap D(z, r)) \leq C r .
$$

The infimum of all such $C$ is called the Carleson norm of $\lambda$, denoted by $\|\lambda\|_{*}$. Let $\Delta$ be the unit disk. In this paper, we mainly focus our attention on the case $\Omega=\Delta$. We denote by $C M(\Delta)$ the set of all Carleson measures on $\Delta$.

We say that a measurable function $\mu(z)$ belongs to $C M^{*}(\Delta)$ if the measure

$$
\frac{|\mu|^{2}}{1-|z|^{2}} d x d y \in C M(\Delta) \text {. }
$$

https://doi.org/10.5186/aasfm.2021.4601

2020 Mathematics Subject Classification: Primary 30F35, 30F60.

Key words: Fuchsian group, Carleson measure, Ruelle's property. 
The importance of the class $C M^{*}(\Delta)$ lies in the fact that it plays a crucial role in the theory of BMOA-Teichmüller space, see $[1,5,8,14]$ etc. If $G$ is a Fuchsian group and $\mu(z)$ a bounded measurable function on $\Delta$ which satisfies

$$
\|\mu(z)\|_{\infty}<1 \quad \text { and } \quad \mu(z)=\mu(g(z)) \overline{g^{\prime}(z)} / g^{\prime}(z)
$$

for every $g \in G$, then we say $\mu$ is a $G$-compatible Beltrami coefficient (or complex dilatation). We denote by $M(G)$ the set of all $G$-compatible Beltrami coefficients. For a $G$-compatible Beltrami coefficient $\mu$, if the measure

$$
\frac{|\mu|^{2}}{1-|z|^{2}} d x d y
$$

is a Carleson measure on $\Delta$, when the Carleson norm is small, then $f_{\mu}(\partial \Delta)$ is a rectifiable (chord-arc) curve, where $f_{\mu}$ is the quasiconformal mapping of the complex plane $\mathbf{C}$ with $i, 1$ and $-i$ fixed, whose Beltrami coefficient equals to $\mu$ a.e. on the unit disk and equals to zero on the outside of the unit disk. This is essential for the proof of the convergence-type first-kind Fuchsian groups failing to have Bowen's property, see [2]. It is also the method to prove that some convergence-type Fuchsian groups fail to have Ruelle's property, see $[12,11]$.

It is important to investigate under which condition the $G$-compatible Beltrami coefficients belong to $C M^{*}(\Delta)$. We call the intersection of $\overline{\mathcal{F}}$ with the unit circle $\partial \Delta$ the boundary at infinity of $\mathcal{F}$, denoted by $\mathcal{F}(\infty)$. In this paper, we first prove:

Theorem 1.1. Let $G$ be a convex cocompact Fuchsian group of the second kind and $\mathcal{F}$ the Dirichlet fundamental domain of $G$ centered at 0 . Let $\mu \in M(G)$ : if there exists a constant $C$ such that, for any $\xi \in \mathcal{F}(\infty)$ (i.e. $\xi$ is in the free edges of $\mathcal{F}$ ) and for any $0<r<1$,

$$
\iint_{B(\xi, r)} \frac{|\mu|^{2} \chi_{\mathcal{F}}}{1-|z|^{2}} d x d y \leq C r
$$

then $\mu$ is in $C M^{*}(\Delta)$, where $\chi_{\mathcal{F}}$ is the characteristic function of the Dirichlet fundamental domain $\mathcal{F}$.

Notice that Theorem 1.1 fails for the case of convex cocompact groups of the first kind (i.e. cocompact groups), since Bowen [6] showed that cocompact groups hold a rigidity property, now called Bowen's property, i.e. the image of the unit circle under any quasiconformal map whose Beltrami coefficient compatible with a cocompact group, is either a circle or has Hausdorff dimension bigger than 1. Hence for any $\mu$ being compatible with cocompact groups, the measure $\frac{|\mu|^{2}}{1-|z|^{2}} d x d y$ is not a Carleson measure.

Furthermore, Theorem 1.1 can be generalized to the finitely generated Fuchsian group of the second kind with some parabolic elements. We have

Theorem 1.2. Let $G$ be a finitely generated Fuchsian group of the second kind with some parabolic elements and $\mathcal{F}$ the Dirichlet fundamental domain of $G$ centered at 0 . Let $\mu \in M(G)$ : if there exists a constant $C$ such that, for any $\xi \in \mathcal{F}(\infty)$ and for any $0<r<1$,

$$
\iint_{B(\xi, r)} \frac{|\mu|^{2} \chi_{\mathcal{F}}}{1-|z|^{2}} d x d y \leq C r
$$

then $\mu$ is in $C M^{*}(\Delta)$. 
This theorem means that the Carleson property of the measures which are compatible with the finitely generated Fuchsian groups can be checked from the points in the set $\mathcal{F}(\infty)$ i.e., the boundary at infinity of the Dirichlet domain $\mathcal{F}$.

Notation. In this paper $\chi_{A}$ always denotes the characteristic function of the set A.

\section{Some lemmas}

The following lemma will be used several times in this paper. I give a short proof here.

Lemma 2.1. Let $\mu$ be a essentially bounded measurable function on $\Delta$. If the measure $\frac{|\mu|^{2}}{1-|z|^{2}} d x d y$ is in $C M(\Delta)$, then there exists a constant $C$ such that, for any $\xi \in \bar{\Delta}$ and all $0<r<2$,

$$
\iint_{B(\xi, r) \cap \Delta} \frac{|\mu|^{2}}{1-|z|^{2}} d x d y \leq C r
$$

where the constant $C$ depends only on the Carleson norm of the measure $\frac{|\mu|^{2}}{1-|z|^{2}} d x d y$ and the essential norm of $\mu$.

Proof. We first choose $0<r<2$ and fix it. For any $\xi \in \bar{\Delta}$, if $\xi \in \partial \Delta$, there is nothing to prove. We suppose $\xi \in \Delta$. If $\operatorname{dist}(\xi, \partial \Delta) \geq 2 r$ (this case only happens when $0<r<0.5)$, where $\operatorname{dist}(\cdot, \cdot)$ denotes the Euclidean distance. Then we have

$$
\iint_{B(\xi, r)} \frac{|\mu|^{2}}{1-|z|^{2}} d x d y \leq \frac{\|\mu\|_{\infty} \pi r^{2}}{1-|1-r|^{2}}=\frac{\|\mu\|_{\infty} \pi r}{2-r} \leq \pi\|\mu\|_{\infty} r .
$$

For the case $\operatorname{dist}(\xi, \partial \Delta) \leq 2 r$, we can choose a point $\eta \in \partial \Delta$ such that $\operatorname{dist}(\eta, \xi)<$ $2 r$. Then we have $B(\xi, r) \subset B(\eta, 4 r)$ and

$$
\iint_{B(\xi, r) \cap \Delta} \frac{|\mu|^{2}}{1-|z|^{2}} d x d y \leq \iint_{B(\eta, 4 r) \cap \Delta} \frac{|\mu|^{2}}{1-|z|^{2}} d x d y \leq 4 C^{*} r,
$$

where $C^{*}$ is the Carleson norm of the measure $\frac{|\mu|^{2}}{1-|z|^{2}} d x d y$.

Hence we let $C=\max \left\{\pi\|\mu\|_{\infty}, 4 C^{*}\right\}$ and the lemma follows.

Remark. By this lemma we see that for any simply connected domain $\Omega \subset \Delta$, If $\frac{|\mu|^{2}}{1-|z|^{2}} d x d y$ is a Carleson measure on $\Delta$, then it is also a Carleson measure on $\Omega$.

In order to prove Theorem 1.1, we will need the following lemma which essentially belongs to Astala and Zinsmeister, see [1], or [2].

Lemma 2.2. For a convergence-type Fuchsian group $G$ and $\mu$ in $M(G)$, if there exists a $0<t<1$ such that the support set of $\mu_{\mathcal{F}}$ is contained in the ball $B(0, t)$ with center 0 and radius $t$. Then $\mu$ is in $C M^{*}(\Delta)$.

For the readers to see more clearly about the property of $\mu$, we give the detail of proof of this lemma here.

Proof. Recall that a sequence $\left\{z_{j}\right\}$ is called an interpolating sequence of $\Delta$ if

(i) $\exists \delta>0, \rho\left(z_{j}, z_{k}\right) \geq \delta$ if $j \neq k$;

(ii) $\sum\left(1-\left|z_{i}\right|^{2}\right) \delta_{z_{i}} \in C M(\Delta)$,

where $\delta_{z}$ stands for the Dirac mass at $z$. 
We first show that the sequence $\{g(0)\}_{g \in G}$ is an interpolating sequence of the unit disk $\Delta$. The sequence $\{g(0)\}_{g \in G}$ satisfies the property (i) of the interpolating sequence immediately from the action of Fuchsian group being discrete. For the property (ii), by a result due to Carleson [7], we know that

$$
\sum_{g \in G}\left(1-|g(0)|^{2}\right) \delta_{g} \in C M(\Delta)
$$

is equivalent to

$$
\inf _{g_{i}} \prod_{g \in G, g \neq g_{i}}\left|\frac{g_{i}(0)-g(0)}{1-\overline{g_{i}(0)} g(0)}\right| \geq \delta>0
$$

In order to show (3.2), it is enough to prove that for any $g_{i} \neq g_{k}$,

$$
\prod_{g \in G, g \neq g_{i}}\left|\frac{g_{i}(0)-g(0)}{1-\overline{g_{i}(0)} g(0)}\right| \equiv \prod_{g \in G, g \neq g_{k}}\left|\frac{g_{k}(0)-g(0)}{1-\overline{g_{k}(0)} g(0)}\right|
$$

Note that

$$
\left|\frac{g_{i}(0)-g(0)}{1-\overline{g_{i}(0)} g(0)}\right|=\tanh 2 \rho\left(g_{i}(0), g(0)\right)
$$

where $\rho\left(g_{i}(0), g(0)\right)$ denotes the hyperbolic distance between $g_{i}(0)$ and $g(0)$. Similarly,

$$
\left|\frac{g_{k}(0)-g(0)}{1-\overline{g_{k}(0)} g(0)}\right|=\tanh 2 \rho\left(g_{k}(0), g(0)\right) \text {. }
$$

Let $\gamma=g_{k} \circ g_{i}^{-1}$, we have $g_{k}=\gamma \circ g_{i}$ and

$$
\begin{aligned}
& \prod_{g \in G, g \neq g_{k}}\left|\frac{g_{k}(0)-g(0)}{1-\overline{g_{k}(0)} g(0)}\right|=\prod_{g \in G, g \neq g_{k}} \tanh \left(2 \rho\left(g_{k}(0), g(0)\right)\right) \\
= & \prod_{g \in G, g \neq g_{k}} \tanh \left(2 \rho\left(\gamma \circ g_{i}(0), g(0)\right)\right)=\prod_{g \in G, g \neq g_{k}} \tanh \left(2 \rho\left(g_{i}(0), \gamma^{-1} \circ g(0)\right)\right) \\
= & \prod_{g \in G, g \neq g_{i}}\left|\frac{g_{i}(0)-g(0)}{1-\overline{g_{i}(0)} g(0)}\right| .
\end{aligned}
$$

Let $g_{i}=i d$ and in this case

$$
\prod_{g \in G, g \neq g_{i}}\left|\frac{g_{i}(0)-g(0)}{1-\overline{g_{i}(0)} g(0)}\right|=\prod_{g \neq i d}|g(0)|=\exp \left(\sum_{g \neq i d} \ln |g(0)|\right) \geq \exp \left(C \sum_{g \neq i d}(1-|g(0)|)\right),
$$

where $C$ is some universal constant.

Thus by the definition of the convergence-type property it follows that the sequence $\{g(0)\}_{g \in G}$ is an interpolating sequence. 
We now prove Lemma 2.2. Suppose the support set of $\mu_{\mathcal{F}}$, denoted by $\operatorname{Supp}\left(\mu_{\mathcal{F}}\right)$ which is contained in the ball $B(0, t)$. For any $\xi \in \partial \Delta$ and $0<r \leq 2$, we have

$$
\begin{aligned}
\iint_{\Delta \cap B(\xi, r)} \frac{|\mu(z)|^{2}}{1-|z|^{2}} d x d y & =\sum_{g \in G} \iint_{g(B(0, t)) \cap B(\xi, r)} \frac{|\mu(z)|^{2}}{1-|z|^{2}} d x d y \\
& =\sum_{g \in G} \iint_{g(B(0, t))} \frac{|\mu(z)|^{2}}{1-|z|^{2}} \chi_{B(\xi, r)} d x d y \\
& \leq \sum_{g \in G}\|\mu\|_{\infty}^{2} \iint_{g(B(0, t))} \frac{1}{1-|z|^{2}} \chi_{B(\xi, r)} d x d y
\end{aligned}
$$

It is easy to see that the hyperbolic radius $t_{\rho}$ of the Euclidean disk $B(0, t)$ is $\ln \frac{1+t}{1-t}$. Hence for any $g \in G$, the disk $g(B(0, t))$ is a hyperbolic disk with center $g(0)$ and hyperbolic radius $t_{\rho}$. By some simple calculation or by [3] we know that the disk $g(B(0, t))$ is contained in the Euclidean disk $B\left(g(0), R_{g}\right)$, where the radius $R_{g}$ is equal to

$$
\frac{(1+|g(0)|)\left(1-e^{t_{\rho}}\right)(1-|g(0)|)}{(1+|g(0)|)+e^{t_{\rho}}(1-|g(0)|)} \leq C(1-|g(0)|),
$$

where $\mathrm{C}$ is some constant depending only on $t$.

Combined with the above discussion, we get

$$
\begin{aligned}
\iint_{\Delta \cap B(\xi, r)} \frac{|\mu(z)|^{2}}{1-|z|^{2}} d x d y & \leq \sum_{g(0) \in B(\xi, r)} \frac{|| \mu||_{\infty} \pi R_{g}^{2}}{1-\left|1-R_{g}\right|^{2}} \\
& \leq C^{\prime} \sum_{g(0) \in B(\xi, r)}(1-|g(0)|) \leq C^{*} r
\end{aligned}
$$

where the constant $C^{*}$ depends only on $C^{\prime}$ and the Carleson norm of the measure $\sum_{g \in G}(1-|g(0)|) \delta_{g(0)}$. Hence the lemma holds.

Remark. In [5], Bishop used the norm property of Schwarzian derivative of holomorphic function under hyperbolic metric to give another proof of Lemma 2.2 for the case of the Beltrami coefficient $\mu$ supported on a compact subset of the surface $\Delta / G$.

A Jordan curve $\gamma$ is said to be a chord-arc curve if there exists a constant $C$ such that for any two points $\xi_{1}, \xi_{2} \in \gamma$, the length of the arc $\gamma_{\xi_{1}, \xi_{2}}$ satisfies

$$
\operatorname{length}\left(\gamma_{\xi_{1}, \xi_{2}}\right) \leq C d\left(\xi_{1}, \xi_{2}\right) \text {, }
$$

where $\gamma_{\xi_{1}, \xi_{2}}$ is the shorter arc of $\gamma$ with endpoints $\xi_{1}, \xi_{2}$ and $d\left(\xi_{1}, \xi_{2}\right)$ means the Euclidean distance between $\xi_{1}$ and $\xi_{2}$.

A result from [15] says that

Lemma 2.3. [15] Let $\Omega$ be a chord-arc domain. Then the following are equivalent:

(a) $d \nu$ is a Carleson measure for $\Omega$.

(b) For $0<p<\infty$, and $f \in H^{p}(\Omega)$,

$$
\iint_{\Omega}|f|^{p} d v \leq C \int_{\partial \Omega}|f|^{p} d s
$$

where $H^{p}(\Omega)=\left\{f:\right.$ is analytic on $\Omega$ and $\left.\int_{\partial \Omega}|f|^{p} d s<\infty\right\}$ and the constant $C$ depends only on the the Carleson norm of $d \nu$. 
Remark. Lemma 2.3 was first given by Carleson [[10],Theorem 3.9, P.61] when $\Omega$ is the upper half plane. Zinsmeister proved that Carleson's theorem remains true for chord-arc domains, see [15].

After this preparatory work, it is time to give the proof of Theorem 1.1.

\section{Proof of Theorem 1.1}

Proof. Let $G$ be a second-kind convex cocompact Fuchsian group and $\mathcal{F}$ be the Dirichlet domain of $G$ with center 0 . Let $\mu$ be an element in $M(G)$. The intersection of the closure of $\mathcal{F}$ with $\partial \Delta$ contains finitely many intervals which are called free edges of $\mathcal{F}$, denoted by $I_{1}, I_{2}, \cdots I_{n}$.

For any $1 \leq i \leq n$, let $q_{i, 1}, q_{i, 2}$ be the endpoints of $I_{i}$. It is well known that both $q_{i, 1}, q_{i, 2}$ do not belong to the limit set. Both sides of $q_{i, j}(j=1$, or 2$)$ are free sides of Dirichlet fundamental domains with different centers.

By the statement of the theorem we know there exists a constant $C$ such that for any $1 \leq i \leq n$, we can choose a ball $B_{i}$ such that $B_{i} \cap \partial \Delta$ contains no limit points of $G$ and $I_{i} \subset B_{i} \cap \partial \Delta$ and for any point $\xi \in I_{i}$ and $0<r<2$,

$$
\iint_{B(\xi, r) \cap \Delta} \frac{|\mu(z)|^{2}}{1-|z|^{2}} \chi_{B_{i} \cap \Delta} d x d y \leq C r
$$

furthermore, the set $\overline{\mathcal{F}}-\bigcup_{i=1}^{n}\left(B_{i} \cap \mathcal{F}\right)$ is compact, denoted by $\mathcal{F}_{c}$.

By Lemma 2.1, we know that the measure

$$
\frac{|\mu(z)|^{2}}{1-|z|^{2}} d x d y
$$

is a Carleson measure on the domain $B_{i} \cap \mathcal{F}$. We divide $\mu$ into two parts. Let

$$
\mu=\sum_{g \in G} \mu \chi_{g\left(\mathcal{F}_{c}\right)}+\sum_{g \in G} \mu \chi_{g(B)}
$$

where $B=\bigcup_{i=1}^{n}\left(B_{i} \cap \mathcal{F}\right)$.

By Lemma 2.2, we know that the measure $\sum_{g \in G} \mu \chi_{g\left(\mathcal{F}_{c}\right)}$ is a Carleson measure on $\Delta$. In the following we only need to show that $\sum_{g \in G} \mu \chi_{B}$ is also a Carleson measure. Without loss of generality, we may assume $\mu=\sum_{g \in G} \mu \chi_{B}$.

Let $\xi$ be an arbitrary point of $\partial \Delta$ and $r$ a positive real number less than 2. In the following we will find a positive constant $C^{*}$ which does not depend on $\xi$ and $r$ such that

$$
\iint_{B(\xi, r) \cap \Delta} \frac{|\mu|^{2}}{1-|z|^{2}} d x d y \leq C^{*} r .
$$

We first consider the following special case: there exists $g \in G$ such that $g(B(\xi, r) \cap$ $\Delta) \subset \mathcal{F}$. By Lemma 2.1 we know that $\frac{|\mu|^{2}}{1-|z|^{2}} d x d y$ is a Carleson measure on the domain $g(B(\xi, r) \cap \Delta)$. Then we have

$$
\begin{aligned}
\iint_{B(\xi, r) \cap \Delta} \frac{|\mu(w)|^{2}}{1-|w|^{2}} d u d v & \leq \iint_{g(B(\xi, r) \cap \Delta)} \frac{\left|\mu\left(g^{-1}(z)\right)\right|^{2}}{1-\left|g^{-1}(z)\right|^{2}}\left|\left(g^{-1}\right)^{\prime}(z)\right|^{2} d x d y \\
& =\iint_{g(B(\xi, r) \cap \Delta)} \frac{\left|\mu\left(g^{-1}(z)\right) \frac{\overline{\left(g^{-1}\right)^{\prime}(z)}}{\left(g^{-1}\right)^{\prime}(z)}\right|^{2}}{1-\left|g^{-1}(z)\right|^{2}}\left|\left(g^{-1}\right)^{\prime}(z)\right|^{2} d x d y \\
& =\iint_{g(B(\xi, r) \cap \Delta)} \frac{|\mu(z)|^{2}}{1-|z|^{2}}\left|\left(g^{-1}\right)^{\prime}(z)\right| d x d y .
\end{aligned}
$$


Since $g$ is a Möbius transformation, the domain $g(B(\xi, r) \cap \Delta)$ is a chord-arc domain. By Lemma 2.3, we have

$$
\begin{aligned}
\iint_{g(B(\xi, r) \cap \Delta)} \frac{|\mu(z)|^{2}}{1-|z|^{2}}\left|\left(g^{-1}\right)^{\prime}(z)\right| d x d y & \leq C_{1} \int_{\partial g(B(\xi, r) \cap \Delta)}\left|\left(g^{-1}\right)^{\prime}(z)\right| d s \\
& =\int_{\partial(B(\xi, r) \cap \Delta)} d s \leq 2 \pi C_{1} r,
\end{aligned}
$$

where the constant $C_{1}$ depends only on the constant $C$ in the statement of the Theorem 1.1. Hence we have

$$
\iint_{B(\xi, r) \cap \Delta} \frac{|\mu(w)|^{2}}{1-|w|^{2}} d u d v \leq 2 \pi C_{1} r
$$

By the above discussion, we easily get that the measure $\frac{|\mu(z)|^{2}}{1-|z|^{2}} d x d y$ is a Carleson measure on $B_{i} \cap \Delta$ for any $1 \leq i \leq n$, since $B_{i} \cap \partial \Delta$ contains no limit points of $G$ and there are finitely many $g_{1}, \cdots, g_{m}$ belonging to $G$ such that

$$
\left(B_{i} \cap \Delta\right) \subset \bigcup_{1}^{m} g_{j}(\mathcal{F}) .
$$

Now we consider the general case. Let $G^{*}$ be the set of all the elements $g$ in $G$ such that $g(B) \cap B(\xi, r) \neq \emptyset$. If $g \in G^{*}$ there are at most three possibilities as follows:

(a) there exist $1 \leq i \leq n, g\left(B_{i} \cap \mathcal{F}\right) \subset B(\xi, r)$;

(b) there exists $1 \leq i \leq n, g\left(B_{i}\right) \cap B(\xi, r) \neq \emptyset$ and $g\left(I_{i}\right) \subset B(\xi, r) \cap \partial \Delta$;

(c) there exist $1 \leq i \leq n, g\left(B_{i}\right) \cap B(\xi, r) \neq \emptyset$ and $g\left(I_{i}\right) \cap B(\xi, r) \cap \partial \Delta \neq \emptyset$.

In case (a), we have

$$
\begin{aligned}
\iint_{g\left(B_{i} \cap \mathcal{F}\right)} \frac{|\mu(w)|^{2}}{1-|w|^{2}} d u d v & \leq \iint_{g\left(B_{i} \cap \Delta\right)} \frac{|\mu(w)|^{2}}{1-|w|^{2}} d u d v \\
& =\iint_{B_{i} \cap \Delta} \frac{\mid \mu\left(\left.g(z) \frac{\overline{g^{\prime}(z)}}{g^{\prime}(z)}\right|^{2}\right.}{1-|g(z)|^{2}}\left|g^{\prime}(z)\right|^{2} d x d y \\
& =\iint_{B_{i} \cap \Delta} \frac{|\mu(z)|^{2}}{1-|z|^{2}}\left|g^{\prime}(z)\right| d x d y \\
& \leq C_{1} \int_{\partial\left(B_{i} \cap \Delta\right)}\left|g^{\prime}(z)\right| d s=C_{1} \int_{\partial g\left(B_{i} \cap \Delta\right)} d s \\
& \leq C_{1} \pi \operatorname{length}\left(g\left(B_{i} \cap \partial \Delta\right)\right),
\end{aligned}
$$

where the second above inequality holds is by Lemma 2.3 and $C_{1}$ depends only on the Carleson norm of $\frac{|\mu(z)|^{2}}{1-|z|^{2}} \mid d x d y$ on $B_{i} \cap \Delta$.

For case (b) we have

$$
\begin{aligned}
\iint_{g\left(B_{i} \cap \mathcal{F}\right) \cap B(\xi, r)} \frac{|\mu(w)|^{2}}{1-|w|^{2}} \mid d u d v & \leq \iint_{g\left(B_{i} \cap \Delta\right) \cap B(\xi, r)} \frac{|\mu(w)|^{2}}{1-|w|^{2}} \mid d u d v \\
& \leq \pi C_{1} \text { length }\left(B_{i} \cap \partial \Delta\right) .
\end{aligned}
$$

For case (c), notice that $g\left(B_{i} \cap \Delta\right) \cap B(\xi, r)$ is a triangle with three circle-arc and the angle corresponding to the side $g\left(B_{i} \cap \partial \Delta\right) \cap B(\xi, r)$ is bigger than some constant, 
we have

$$
\operatorname{length}\left(\partial\left(g\left(B_{i} \cap \Delta\right) \cap B(\xi, r)\right)\right) \leq C_{2} \operatorname{length}\left(g\left(B_{i} \cap \partial \Delta\right) \cap B(\xi, r)\right) .
$$

where the constant $C_{2}$ depends only on the Carleson norm of $\frac{|\mu(z)|^{2}}{1-|z|^{2}} d x d y$ on $B_{i} \cap \Delta$ and the angle between $\partial B_{i}$ and $\partial \Delta$.

By a similar discussion as case(a) we have

$$
\iint_{g\left(B_{i} \cap \mathcal{F}\right)} \frac{|\mu(w)|^{2}}{1-|w|^{2}} d u d v \leq \pi C_{2} \operatorname{length}\left(g\left(B_{i} \cap \partial \Delta\right) \cap B(\xi, r)\right) .
$$

Since for every $1 \leq i \leq n$, the arc $B_{i} \cap \partial \Delta$ does not contain the limit points of $G$. Hence for $g_{1}, g_{2} \in G^{*}$ if $g_{1}\left(B_{i}\right) \cap B(\xi, r) \neq \emptyset$ and $g_{2}\left(B_{i}\right) \cap B(\xi, r) \neq \emptyset$, the images of $B_{i} \cap \partial \Delta$ under maps $g_{1}, g_{2}$, respectively, do not overlap. Hence we have

$$
\begin{aligned}
& \iint_{B(\xi, r) \cap \Delta} \frac{|\mu(w)|^{2}}{1-|w|^{2}} d u d v \leq \pi C^{*} \sum_{g \in G^{*}} \operatorname{length}(g(B) \cap B(\xi, r) \cap \partial \Delta) \\
& \leq \pi C^{*} \operatorname{length}(B(\xi, r) \cap \partial \Delta) \leq 2(\pi)^{2} C^{*} r,
\end{aligned}
$$

where $C^{*}$ equals to the maximum value of the constants which appeared in the proof of this theorem and $B=\bigcup_{i}^{n}\left(B_{i} \cap \Delta\right)$. This completes the proof.

\section{Proof of Theorem 1.2}

Proof. Let $G$ be a finitely generated Fuchsian group of second kind with some parabolic elements. Since the generator of $G$ contains finite elements, without loss of generality, we may suppose that the generator of $G$ contains only one parabolic element $\gamma$ and suppose $\xi \in \mathcal{F}(\infty)$ be the fixed point of the parabolic element $\gamma$.

We divide $\mu$ into two parts. Let

$$
\mu=\sum_{g \in G} \mu \chi_{g\left(\mathcal{F}^{*}\right)}+\sum_{g \in G} \mu \chi_{(g(B \cap \mathcal{F}))},
$$

where $\mathcal{F}^{*}=\mathcal{F}-(B \cap \mathcal{F})$ and $B$ is a disk with center $\xi$ and radius $r_{0}$ (that is sufficiently small such that $\partial B$ intersect with the sides of $\mathcal{F}$ which have $\xi$ as a common vertex). Let $\gamma_{0}$ be the arc of $\partial B$ between the sides of $\mathcal{F}$ which have $\xi$ as a common vertex.

By Theorem 1.1, we know that the measure $\sum_{g \in G} \mu \chi_{g\left(\mathcal{F}^{*}\right)}$ is a Carleson measure on $\Delta$. In the following we only need to show that $\sum_{g \in G} \mu \chi_{g(B \cap \mathcal{F})}$ is also a Carleson measure. Without loss of generality, we may assume $\mu=\sum_{g \in G} \mu \chi_{g(B \cap \mathcal{F})}$.

We first show that the hyperbolic area of $B \cap \mathcal{F}$ is finite.

By a conformal mapping $\varphi(z)$ which maps the unit disk $\Delta$ onto the upper half plane $\mathbf{H}$, we only need to show that the hyperbolic area of the image $\varphi(B \cap \mathcal{F})$ is finite, since hyperbolic area is unchanged under conformal mapping. Without loss of generality, we may suppose $\varphi(\xi)=0$. The images of the sides of $\mathcal{F}$ with $\xi$ as a vertex under the mapping $\varphi$ are contained in two circles, denoted by $C_{1}$ and $C_{2}$, respectively. Let $C_{1}$ be the circle

$$
\left(x-r_{1}\right)^{2}+y^{2}=r_{1}^{2}
$$

and $C_{2}$ the circle

$$
\left(x+r_{2}\right)^{2}+y^{2}=r_{2}^{2} .
$$

The tangent point of $C_{1}$ and $C_{2}$ is 0 , see Figure 1 . The images of the arc $\gamma_{0}$ is contained in a circle with center 0 and radius $r_{3}$. 
Then we have

$$
\iint_{B \cap \mathcal{F}} \frac{1}{(1-|z|)^{2}} d x d y=\iint_{\varphi(B \cap \mathcal{F})} \frac{1}{4 v^{2}} d u d v=\int_{0}^{r_{3}} d r \int_{\arccos \left(\frac{r}{2 r_{1}}\right)}^{\arccos \left(\frac{-r}{2 r_{2}}\right)} \frac{1}{4 r \sin ^{2} \theta} d \theta .
$$

It is easy to see that the limit

$$
\lim _{r \rightarrow 0} \int_{\arccos \left(\frac{r}{2 r_{1}}\right)}^{\arccos \left(\frac{-r}{2 r_{2}}\right)} \frac{1}{4 r \sin ^{2} \theta} d \theta=\frac{1}{8}\left(\frac{1}{r_{2}}+\frac{1}{r_{1}}\right) .
$$

Hence by some easy calculation, the hyperbolic area of $B \cap \mathcal{F}$ is finite.

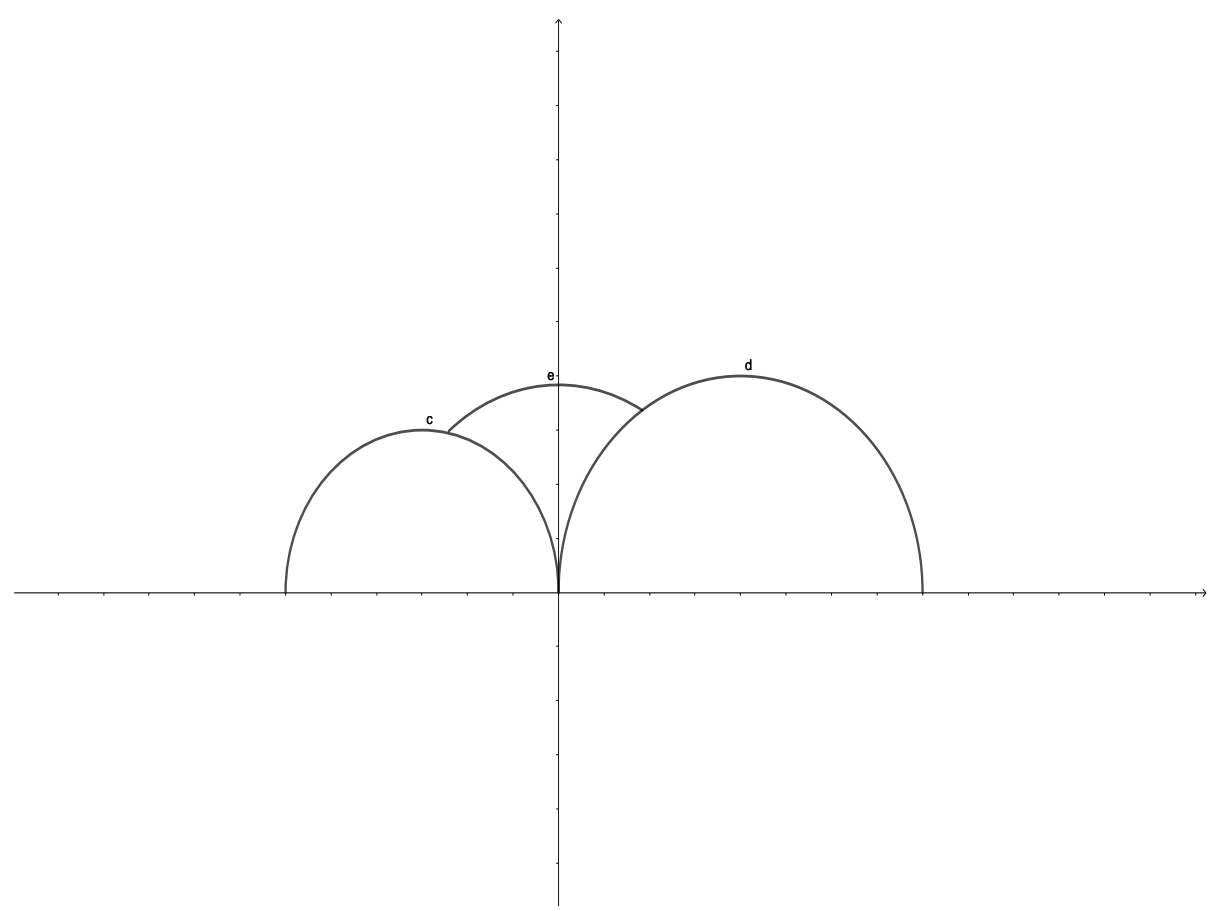

Figure 1.

We continue to prove Theorem 1.2. For any $g \in G$, we have

$$
\begin{aligned}
\iint_{g(B \cap \mathcal{F})} \frac{|\mu(w)|^{2}}{1-|w|^{2}} d u d v & \leq \iint_{g(B \cap \mathcal{F})} \frac{1}{1-|w|^{2}} d u d v \\
& =\iint_{B \cap \mathcal{F}} \frac{\left|g^{\prime}(z)\right|^{2}}{1-|g(z)|^{2}} d x d y \\
& =\iint_{B \cap \mathcal{F}} \frac{1}{1-|z|^{2}}\left|g^{\prime}(z)\right| d x d y \\
& =\iint_{B \cap \mathcal{F}} \frac{1-|g(z)|^{2}}{\left(1-|z|^{2}\right)^{2}} d x d y \\
& \leq C_{1}\left(1-\left|g\left(z_{0}\right)\right|\right) \iint_{B \cap \mathcal{F}} \frac{1}{\left(1-|z|^{2}\right)^{2}} d x d y \\
& \leq C\left(1-\left|g\left(z_{0}\right)\right|\right),
\end{aligned}
$$

where $z_{0}$ is any point in $\gamma_{0}, C_{1}$ depends on $z_{0}$ and the hyperbolic length of $\gamma_{0}$, and $C$ depends on $C_{1}$ and the hyperbolic area of $B \cap \mathcal{F}$. The fourth above equality holds since

$$
\frac{\left|g^{\prime}(z)\right|}{1-|g(z)|^{2}}=\frac{1}{1-|z|^{2}} \quad \text { for any } g \in G
$$


and the last second above inequality holds since the hyperbolic length of $\gamma_{0}$ is finite.

Let $\eta$ be any point on the unit circle and $B(\eta, r)$ the disk with center $\eta$ and radius $r$, here $0<r<1$. By the proof of Lemma 2.2, we know that the sequence $\left\{g\left(z_{0}\right)\right\}_{g \in G}$ is an interpolating sequence. Hence the images of $B \cap \mathcal{F}$ under $g \in G$ which are contained in the disk $B(\eta, r)$ satisfy

$$
\iint_{B \cap \Delta} \frac{|\mu(w)|^{2}}{1-|w|^{2}} d u d v \leq \sum_{g \in G, g(B \cap \mathcal{F}) \cap B(\eta, r) \neq \emptyset} \iint_{g(B \cap \mathcal{F})} \frac{|\mu(w)|^{2}}{1-|w|^{2}} d u d v \leq C r,
$$

which completes the proof of the theorem.

In [4], Bishop showed that all divergence type Fuchsian groups have Bowen's property, hence Theorem 1.1 fails for the case of divergence-type groups. By Lemma 2.2 we know that for all convergence-type Fuchsian groups with compact support Beltrami coefficient, the result also holds. We ask the following question: does the result holds for all convergence-type Fuchsian groups?

\section{Some applications}

In this section we give an application of Theorem 1.1 and 1.2. In [1, p. 617, Theorem 7], Astala and Zinsmeister showed that there exist Fuchsian groups $G$ of the second kind such that the Hausdorff dimension of the quasicircle $f(\partial \Delta)$ is not a real analytic function on Teichmüller space $T(G)$. In [11], the author and Wu Shengjian showed that the result holds for any second kind Fuchsian groups. By Theorem 1.1 and Theorem 1.2, we can give a very short proof of the result for the case of finitely generated Fuchsian groups of second kind. We have

Corollary 5.1. Let $G$ be any finitely generated Fuchsian groups of the second kind with or without parabolic elements, the Hausdorff dimension of the quasicircle $f(\partial \Delta)$ is not a real analytic function on Teichmüller space $T(G)$.

Proof. We will construct a $\mu \in C M^{*}(\Delta) \cap M(G)$ such that the Hausdorff dimension of the quasicircle $f_{\mu}(\partial \Delta)$, denoted by $\operatorname{dim}\left(f_{\mu}(\partial \Delta)\right)$, is bigger than 1 . Let $\mu^{*} \in C M(\Delta)$ such that $\operatorname{dim}\left(f_{\mu^{*}}(\partial \Delta)\right)>1$, without loss of generality we may suppose $\operatorname{dim}\left(\partial f_{\mu^{*}}(\overline{\mathcal{F}} \cap \partial \Delta)\right)>1$ (the existence of such a $\mu^{*}$ can be found in $([1$, p. 624] $)$.

Let $\mu_{\mathcal{F}}=\mu^{*}(z)$ for $z \in \mathcal{F}$. Now we translate $\mu_{\mathcal{F}}$ by the group $G$ to the whole disk $\Delta$. We get

$$
\mu(z)= \begin{cases}\mu^{*}(z), & z \in \mathcal{F} \\ \mu^{*}(g(z)), & g(z) \in \mathcal{F}, g \in G .\end{cases}
$$

By Theorem 1.1 or Theorem 1.2, we can see that $\mu \in C M^{*}(\Delta) \cap M(G)$. Now we consider the mapping $\psi: t \rightarrow \operatorname{dim}\left(f_{t \mu}\right)$. By [13] we know that there exist a $\varepsilon>0$ such that, for all $|t|<\varepsilon$, the quasicircle $f_{t \mu}(\partial \Delta)$ is a rectifiable curve. This implies that $\psi$ can not be real analytic on $\Delta$.

Acknowledgements. It is my pleasure to thank professor Michel Zinsmeister for inviting me to the University of Orleans as a visiting scholar for one year and for some discussions on topics related to this paper. This work was done during my visit in Orléans. The author would also like to thank China Scholar Council for life-expenses in Orléans. This work was supported by the Science and Technology Development Fund of Tianjin Commission for Higher Education (Grant No. 2017KJ095). The author would also like to thank the refrees' valuable suggestions and several corrections which greatly improves the presentation of the paper. 


\section{References}

[1] Astala, K., and M. Zinsmeister: Teichmüller spaces and BMOA. - Math. Ann. 289, 1991, 613-625.

[2] Astala, K., and M. Zinsmeister: Rectifiability in Teichmüller theory. - In: Topics in Complex Analysis, Banach Center Publications 31, 1995, 45-52.

[3] Beardon, A. F.: The geometry of discrete group. - Springer-Verlag, 1983.

[4] Bishop, C. J.: Divergence groups have the Bowen property. - Ann. of Math. 154, 2001, 205217.

[5] Bishop, C. J.: Compact deformations of Fuchsian group. - J. Anal. Math. 87, 2002, 5-36.

[6] Bowen, R.: Hausdorff dimension of quasicircles. - Publ. Math. Inst. Hautes. Études. Sci. 50, 1979, 11-25.

[7] Carleson, L.: An interpolation problem for bounded analytic functions. - Amer. J. Math. 4, 1958, 921-930.

[8] CuI, G.: Integrably asymptotic affine homeomorphisms of the circle and Teichmüller spaces. Sci. China Ser. A 43, 2000, 267-279.

[9] Dal’Bo, F.: Geodesic and horocyclic trajectories. - Springer, 2011.

[10] Garnett, J. B.: Bounded analytic functions. - Academic Press, New York, 1981.

[11] Huo, S., and S. Wu: The failure of analyticity of Hausdorff dimensions of quasi-circles of Fuchsian groups of the second kind. - Proc. Amer. Math. Soc. 143, 2015, 1101-1108.

[12] Huo, S., and M. Zinsmerster: On Ruelle's property. - Preprint, arXiv:1906.01291v1.

[13] Pommerenke, Ch.: Schlichte Functionen und BMOA. - Comment. Helv. 52, 1977, 591-602.

[14] Shen, Y., and H. Wei: Universal Teichmüller space and BMO. - Adv. Math. 234, 2013, $129-148$.

[15] Zinsmeister, M.: Les domaines de Carleson. - Michigan Math. J. 36:2, 1989, 213-220.

Received 25 June $2019 \bullet$ Accepted 8 January 2020 